\title{
84th Annual Meeting of the Swiss Society for Dermatology and Venereology
}

October 31 to November 2, 2002, Bern

Editor: Prof. L.R. Braathen

\section{Contents}

Lichen striatus in Adults or

'Adult Blaschkitis'?

There Is No Need for a New Naming

T. Hofer

Clinical and Therapeutic

Aspects of Polymorphous

Light Eruption

R. Dummer, K. Ivanova,

E.P. Scheidegger, G. Burg

\section{Effect of High-Dose}

Intravenous Immunoglobulin

Therapy in Stevens-Johnson

Syndrome: A Retrospective,

Multicenter Study

C. Prins, C. Vittorio, R.S. Padilla,

T. Hunziker, P. Itin, J. Förster,

E.-B. Bröcker, J.-H. Saurat,

L.E. French
Anaphylaxis to the

Carbohydrate

Carboxymethylcellulose in

Parenteral Corticosteroid

Preparations

P.L. Bigliardi, J. Izakovic, J.M. Weber,

A.J. Bircher . . . . . . . 100

Decreased CD44 Expression and Stromal Hyaluronate

Accumulation in Myxoid

\section{Dermatofibroma}

E. Çalıkoglu, P. Chavaz, J.-H. Saurat,

G. Kaya

Posttransplant CD30+

Anaplastic Large Cell

Lymphoma with Skin and

Lymph Node Involvement

H. Yurtsever, W. Kempf,

R.H. Laeng
Unusual Manifestation of Specific Cutaneous Involvement by B-Cell Chronic Lymphocytic Leukemia:

Spontaneous Regression with Scar Formation

D.V. Kazakov, I.E. Belousova,

S. Michaelis, G. Palmedo,

A.V. Samtsov, W. Kempf

Imiquimod Induces Complete Clearance of a PUVA-Resistant Plaque in Mycosis fungoides

R. Dummer, M. Urosevic, W. Kempf,

D. Kazakov, G. Burg ～. . . . . . . . 116

Treatment of Verrucous Carcinoma with Imiquimod and $\mathrm{CO}_{2}$ Laser Ablation

L.M. Heinzerling, W. Kempf, J. Kamarashev, J. Hafner,

F.O. Nestle

\section{KARGER @ 2003 S. Karger AG, Basel}

